\title{
La patologia ossea nel paziente trapiantato renale
}

\section{S. Bandini}

U. O. Nefrologia, Dialisi e Trapianto, Azienda Ospedaliera Careggi, Firenze

La patologia ossea del trapiantato renale rappresenta uno dei problemi clinici più importanti che il nefrologo deve affrontare: il 91\% dei pazienti trapiantati renali ha evidenza istologica di osteodistrofia ed osteopenia, la perdita di massa ossea è precoce nel I periodo post-trapianto e l'incidenza di fratture è assai elevata. Il trapianto renale è una terapia efficace, in grado di risolvere molte alterazioni metaboliche del paziente uremico, ma corregge solo parzialmente l'osteodistrofia renale che spesso evolve e interagisce con una osteopatia secondaria a fattori connessi con il trapianto. A fattori di rischio pre-trapianto si associano fattori di rischio post-trapianto che "insistono sull'osso del paziente trapiantato renale" e che ne condizionano il quadro istopatologico e l'efficacia di un'adeguata prevenzione e trattamento (1-4) (Tab. I).

\section{Aspetti patogenetici dell'osteopatia del trapianto}

Molteplici, come si evince dalla Tabella I sono i meccanismi patogenetici che "sottendono" all'osteopatia del trapiantato: in estrema sintesi a fattori responsabili di un ridotto turn over osseo si associano fattori responsabili di

TABELLA I - OSTEOPATIA DEL PAZIENTE TRAPIANTATO RENALE. PRINCIPALI FATTORI DI RISCHIO PRETRAPIANTO

1) Malattia adinamica dell'osso

2) Iperparatiroidismo

3) Trattamento farmacologico (steroidi, farmaci immunosoppressori)

4) Malnutrizione

5) Ipogonadismo

\section{PRINCIPALI FATTORI DI RISCHIO POST-TRAPIANTO}

1) Iperparatiroidismo persistente

2) Alterazioni del metabolismo minerale: ipofosfatemia, ipercalcemia

3) Ridotta funzione renale, acidosi metabolica

4) Terapia immunosoppressiva: steroidi-ciclosporina-tacrolimus

5) Età

6) Diabete 
aumentato riassorbimento osseo: ne deriva un ridotto volume osseo, un quadro ingravescente di osteopeniaosteoporosi con aumentato rischio di frattura anche per traumi di modesta entità (Figg.1, 2).

Le forme più frequenti di patologia ossea che il nefrologo deve trattare sono: l'osteopatia ad alto turnover (l'iperparatiroidismo persistente del trapiantato renale associato o meno a ipercalcemia), la cosiddetta sindrome osteopenica-osteoporotica, l'osteonecrosi asettica, gli eventi fratturanti, oltre ad alcune alterazioni del metabolismo minerale caratteristiche del paziente trapiantato (ipercalcemia, ipofosfatemia, acidosi metabolica) $(5,6)$.

\section{Iperparatiroidismo (IPT) persistente}

Dopo un trapianto renale ben funzionante si ha nel tempo normalizzazione della sintesi di calcitriolo, riduzione (in tempi più rapidi) dei livelli fosforemici e un adeguato controllo della funzione paratiroidea: si assiste a un declino iniziale dei livelli di PTH, ma esistono molte incertezze sui tempi necessari per la normalizzazione della funzione paratiroidea nel tempo. Alcuni autori riferiscono di una normalizzazione dei livelli di PTH entro un anno, altri, per contro, della persistenza di elevati livelli di PTH per anni in una larga percentuale di pazienti $(30,40 \%)(7-9)$. Montalban in una review recente evidenzia come oltre il $40 \%$ dei trapiantati renali presenta un quadro di iperparatiroidismo a 10 anni dal trapianto con livelli di creatinina paranormali (10). Tale evenienza dipende in molti casi dalla persistenza di un IPT residuale (associato nel 30-50\% dei casi a ipercalcemia, i casi più difficili da trattare) correlata alla lenta involuzione del tessuto paratiroideo iperplastico, al grado di iperplasia ghiandolare (proliferazione mono-

\section{Post-transplantation Bone Disease}

\author{
Decreased bone turn-over \\ $+$ \\ Normal-High bone resorption
}

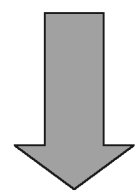

Uncoupling bone formation and resorption

Fig. 1 clonale + iperplasia nodulare) all'età dialitica pre-trapianto e anche alla funzione dell'organo trapiantato. Pazienti, quindi, che non raggiungono un VFG adeguato, sono a grande rischio di sviluppare un IPT persistente post-trapianto, così come quelli che pur raggiungendo una funzione renale normale sviluppano nel tempo una progressiva perdita della funzione renale a seguito di un rigetto cronico.

Se una severa ipercalcemia, espressione di un iperparatiroidismo di grave entità pre-trapianto, compare nei primi giorni post-trapianto, associata a una tardiva ripresa della funzione renale si può verificare un quadro tubulo necrosi acuta e una rapida e diffusa comparsa di calcificazioni sottocutanee e vascolari con fenomeni gravi di calcifilassi, complicanze cardiovascolari che possono addirittura mettere a rischio la vita del paziente (8). Tali eventi sono rari e sono espressione di un mancato o non adeguato controllo di un IPT severo pre-trapianto. Va tenuto presente che la migliore prevenzione dell'IPT residuale è un rigoroso trattamento medico o, nei casi più gravi, chirurgico dei pazienti iperparatiroidei in lista di attesa per trapianto. Molto più spesso il nefrologo deve trattare un iperparatiroidsmo "persistente" ipercalcemico post-trapianto, in presenza di una adeguata funzione renale che può provocare nel tempo una riduzione della massa minerale ossea e una insufficienza funzionale tardiva dell'organo trapiantato. È opportuno, in questi casi seguire clinicamente il paziente con esami biochimici frequenti (PTH, calcemia, fosforemia, fosfatasi alcalina ecc.) e strumentali adeguati (ecografia del collo, scintigrafia paratiroidea) senza affrettare una opzione chirurgica: i pazienti con IPT persistente sono, infatti, generalmente asintomatici e di norma l'ipercalcemia tende a risolversi spontaneamente in un periodo variabile da pochi mesi a qualche anno $(5,9)$. Le 2 maggiori indicazioni alla para-

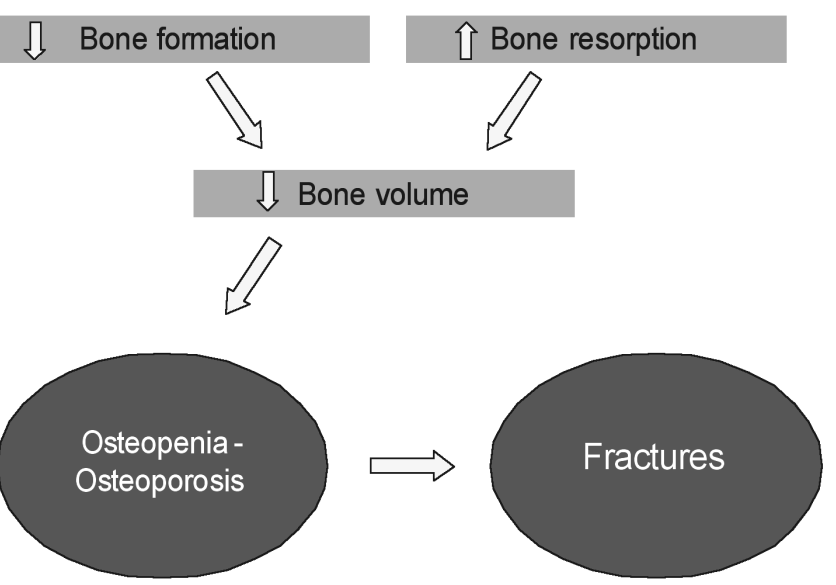

Fig. 2 
tiroidectomia ( $5 \%$ dei pazienti trapiantati) sono: una ipercalcemia precoce sintomatica $(>12.5 \mathrm{mg} / \mathrm{dL})$ come precedentemente descritto, o una ipercalcemia asintomatica con livelli elevati di PTH dopo un anno o più dal trapianto, associata a riduzione della funzione renale non dipendente da altra causa.

Trattamento chirurgico dell'IPT persistente ipercalcemico. La paratiroidectomia comunque non è esente da problemi e prima di porne l'indicazione va considerato che sono stati segnalati anche recentemente eventi avversi quali: una riduzione della funzione renale, una ipocalcemia resistente al trattamento farmacologico e la comparsa di una patologia ossea a basso turnover aggravata ulteriormente dal trattamento immunosoppressore. Trattamento farmacologico dell'IPT persistente ipercalcemico. Come è ben intuibile in questi pazienti il trattamento con vitamina $\mathrm{D}$ e calcio non è proponibile eccetto che nei pazienti con IPT normocalcemici. Del tutto recentemente però, nel trattamento dell'IPT ipercalcemico post-trapianto, sono stati utilizzati i calciomimetici (tali farmaci attualmente in Italia sono indicati solo per il trattamento dell'IPT del paziente in uremia stadio 5) con buoni risultati nel ridurre i livelli di PTH e della calcemia anche se sono stati segnalati casi di intolleranza gastrointestinale e di lieve riduzione della funzione renale. A questo proposito, è stato, del tutto recentemente, evidenziato come gli effetti del calciomimetico sulla ghiandola paratiroidea persistono con la sospensione del farmaco e si ripristinano i livelli di creatinina pretrattamento. Allo stato attuale pertanto, pur considerando che gli studi proposti hanno presentato casistiche limitate e brevi periodi di follow-up, i calciomimetici, potrebbero rappresentare l'unico vero ausilio farmacologico del nefrologo nel trattamento dell'IPT post trapianto (11-17).

\section{Alterazioni del metabolismo minerale}

\section{IPOFOSFATEMIA}

L’ipofosfatemia è un'alterazione metabolica frequente nel paziente trapiantato renale ed è dovuta a una eccessiva escrezione urinaria di fosfati. Si verifica in circa il $50-80 \%$ dei pazienti nei primi 3 mesi del trapianto: dopo un anno dal trapianto meno del $5 \%$ dei pazienti presenterà ipofosfatemia. Liperfosfaturia è di origine multifattoriale: alti livelli di PTH, bassi livelli di calcitriolo, la somministrazione di diuretici, il trattamento steroideo e alti livelli di una fosfatonina di recente scoperta, il Fibroblastic Grow Factor 23, possono determinare questa alterata regolazione del trasporto di fosfati a livello del tubulo renale. Più precisamente, la fosfaturia sarebbe secondaria a un deficit del sistema di trasporto del fosfato Nadipendente presente a livello del tubulo prossimale (NaPi2a: cotra- sportatore sodio/fosforo). Il FGF 23, identificato a seguito di studi sul rachitismo ipofosfatemico autosomico dominante, è una proteina di origine osteoblastica, i cui livelli sierici sono elevati sia nella IRC che nei primi mesi post-trapianto. Questa proteina agirebbe, indipendentemente dal PTH, a livello del tubulo prossimale renale, mediante recettori specifici FGFR (FGFReceptor): un cofattore identificato nella proteina Klotho (indispensabile per il legame del FGF23 al recettore) permette di esercitare l'effetto biologico di inibizione del cotrasportatore sodio/fosforo NaPi2a (18-21).

Una grave ipofosfatemia $(\mathrm{P}<0.9 \mathrm{mg} / \mathrm{dL})$ può determinare anemia emolitica, rabdomiolisi, osteopenia-osteomalacia, complicanze neurologiche, insufficienza respiratoria, aritmie e scompenso cardiocircolatorio. È necessario pertanto, per evitare tali temibili complicanze, la supplementazione di fosfati per os in presenza di livelli di fosforemia inferiori a $2.5 \mathrm{mg} / \mathrm{dL}$. Il dipiridamolo, farmaco che aumenta l'assorbimento tubulare dei fosfati, è stato utilizzato con successo da Balal et al nel trattamento della iperfosforemia dei pazienti trapiantati renali (22).

\section{IPERCALCEMIA}

L'ipercalcemia è un evento comune dopo trapianto renale: essa è legata in primis, come già accennato, alla persistenza di un iperparatiroidismo pretrapianto. Esistono comunque altre cause di ipercalcemia nel paziente trapiantato renale: 1) l'immobilità postoperatoria; 2) l'ipofosfatemia; 3) il riassorbimento del fosfato di calcio dai depositi extraossei con risoluzione delle calcificazioni; 4) la progressiva normalizzazione dei livelli sierici di calcitriolo. A questo proposito in presenza di una normale funzione renale post-trapianto, si assiste a un recupero dell'attività calcemizzante del PTH che si traduce direttamente, in un aumentato riassorbimento di calcio dall'osso e a livello renale (tubulo distale) e indirettamente in un aumentato assorbimento intestinale di calcio, calcitriolo dipendente. Lipercalcemia da IPT usualmente si risolve, in presenza di un'adeguata funzione renale, con la normalizzazione della funzione della ghiandola paratiroidea e il ripristino di livelli normali di vitamina $\mathrm{D}$. In una percentuale modesta di casi (1-5\%) l'ipercalcemia persiste espressione di una insufficiente e molto lenta involuzione del tessuto paratiroideo iperplastico. In tali casi può essere indicata la PTX.

\section{ACIDOSI METABOLICA}

Molteplici cause nel paziente trapiantato renale determinano acidosi metabolica: una acidosi tubulare renale, una persistente uremia, una chetoacidosi diabetica secondaria a diabete mellito post-trapianto ecc. L'effetto tampone dell'osso come compenso dell'acidosi, determina una ul- 
teriore perdita di massa ossea con aggravamento del quadro osteopenico. È indispensabile pertanto un controllo di routine dell'equilibrio acido-base per correggere se necessario questo difetto metabolico.

\section{Trattamento immunosoppressore e patologia ossea del trapiantato renale}

La sindrome osteopenica-osteoporotica, le fratture secondarie alla patologia osteoporotica, l'osteonecrosi (necrosi avascolare) sono le complicanze ossee più strettamente correlate al trapianto.

\section{SINDROME OSTEOPENICA-OSTEOPOROTICA}

Il fattore patogenetico di maggior rilievo della sindrome osteopenica-osteoporotica caratteristica patologia ossea del paziente trapiantato, è rappresentato dal trattamento immunosoppressore, ma un ruolo non secondario hanno anche altri fattori quali: il sesso, l'età, la malattia diabetica, la terapia pregressa con corticosteroidi, un iperparatiroidismo severo e, in particolare, la presenza di una patologia ossea a basso turnover (malattia adinamica dell'osso) pretrapianto. L'Adinamic Bone Disease rappresenta, infatti, attualmente l'osteopatia più frequente nel paziente uremico. $\mathrm{E}$ caratterizzata istologicamente da un ridotto volume osseo associato a un ridotto numero e attività degli osteoblasti, degli osteoclasti e da un franco ipoparatiroidismo. Alcuni autori hanno evidenziato nel paziente uremico una correlazione positiva tra malattia adinamica dell'osso (ABD), bassi livelli di PTH e un aumentato rischio di fratture. Ne derivano due considerazioni: 1) un trattamento immunosoppressore se effettuato in un paziente con $\mathrm{ABD}$ può accentuare la demineralizzazione dell'osso, accelerando la comparsa di una grave osteoporosi con notevole rischio di fratture $(3-6) ; 2)$ per contro un iperparatiroidismo di lieve entità, per lo stimolo esercitato dal paratormone sul turnover osseo, potrebbe giocare un ruolo protettivo sulla evoluzione della patologia ossea propria del trapiantato renale (23).

Studi prospettici hanno dimostrato un rapido decremento della massa ossea post-trapianto: è coinvolto in modo prevalente l'osso trabecolare (rachide lombare) ma è interessato anche l'osso corticale (collo del femore). Alcuni autori riferiscono di una riduzione del 10\% della densità minerale ossea (BMD) durante i primi 6 mesi dopo il trapianto. Altri studi hanno evidenziato un decremento della densità minerale ossea di 1.6\%/mese a livello della colonna lombare durante i primi 5 mesi dopo il trapianto. La massa ossea tende a stabilizzarsi entro il $12^{\circ}-18^{\circ}$ mese, in alcuni casi, con lieve miglioramento ma se non si adottano opportuni provvedimenti terapeutici, la riduzione del contenuto minerale osseo può pro- gredire. A tal proposito, studi longitudinali effettuati in pazienti trapiantati renali da lungo tempo hanno dimostrato una lenta ma significativa perdita di BMD: 1.7\% /anno (2-6).

La rapida perdita di massa ossea dopo trapianto renale è secondaria per lo più al trattamento immunosoppressore e segnatamente steroideo. Il grado di riduzione della densità minerale dell'osso è direttamente correlato alla dose e alla durata della somministrazione di steroidi. Gli steroidi possiedono molteplici azioni osteopenizzanti:

a) inibiscono la "bone formation" riducendo il reclutamento e la differenziazione delle cellule osteoblastiche e inducendo apoptosi degli osteoblasti maturi;

b) inducono un aumentato riassorbimento osseo sia mediante azione diretta di stimolo sulla osteoclastogenesi dalle cellule progenitrici all'osteoclasta maturo (mediante attivazione sull'osteoblasta, tramite il RANKL (ligando per il RANK) del recettore RANK presente sui progenitori delle cellule osteoclastiche) sia mediante una diretta inibizione degli ormoni steroidei gonadici (Figg. 1, 2);

c) inibiscono la sintesi renale di $1,25(\mathrm{OH})_{2} \mathrm{D} 3$ cui consegue un ridotto assorbimento intestinale di calcio, una ipocalcemia che stimola un'aumentata secrezione di PTH con incremento del "bone resorption";

d) inibiscono infine alcune citochine, che favoriscono l'attività osteoblastica (ad esempio: l'insuline-like growth factor-1 ecc.) (24-30).

Gli effetti delle altre terapie immunosoppressori sull'osso non sono state ancora chiaramente stabilite. Come è ben noto ciclosporina (Csa) e tacrolimus (FK-506) inibiscono la funzione delle cellule $T$ e sono assai efficaci nel ridurre gli episodi di rigetto post-trapianto. La ciclosporina ha mostrato azioni non univoche sul metabolismo osseo: sperimentalmente sui ratti ha determinato un quadro di osteopenia, aumentando il riassorbimento osseo; ma studi condotti in vitro su linee cellulari dell'osso dimostrerebbero una inibizione del riassorbimento osseo mediante riduzione della formazione di osteoclasti maturi. Similmente, gli studi condotti sull'uomo si presentano con dati contrastanti: secondo alcuni autori il trattamento a lungo termine con sola ciclosporina in trapiantati renali avrebbe un'azione francamente osteopenizzante, secondo altri il trattamento con ciclosporina da sola senza associazione con steroidi non sarebbe associato a una significativa demineralizzazione ossea. Il tacrolimus causa come la ciclosporina osteopenia nei ratti. Sono limitati gli studi sugli effetti del tacrolimus sull' osso del paziente trapiantato renale. Sembrerebbe che il tacrolimus associato ai corticosteroidi, permettendo una riduzione della dose di steroide maggiore che con la ciclosporina, possedesse un minor effetto osteopenizzate rispetto alla ciclosporina. 
Non è stata studiata adeguatamente l'azione degli altri farmaci immunosoppressori (sirolimus, micofenolato, azatioprina) sul metabolismo osseo: essi avrebbero comunque un modesto effetto sulla massa ossea (31-35). È opportuno riferire di alcuni dati istopatologici della patologia ossea post-trapianto derivanti da indagini bioptiche. Tali informazioni derivano da pochi studi, le casistiche sono assai limitate e i reperti bioptici spesso descrivono lesioni ossee eterogenee che possono essere espressione di una patologia ossea ad alto turnover, esito di un iperparatiroidismo persistente (aumento del BFR e incremento dell'indice osteoclastico) o per contro e più frequentemente, di una patologia a basso turnover con reperti di osteopenia/osteoporosi ${ }^{1}$ e/o di osteomalacia/osso adinamico con reperti istomorfometrici caratteristici: riduzione del BFR (bone formation rate) e allungamento dei tempi di mineralizzazione ${ }^{2}$ associati a normale attività di riassorbimento osseo. Recenti lavori hanno confermato che una alterazione della osteoblastogenesi e l'apoptosi delle cellule osteoblastiche sono fra i principali determinanti della precoce perdita di massa ossea post-trapianto, ma hanno anche evidenziato come livelli di PTH moderatamente sopra la norma potrebbero avere un ruolo "protettivo" sulla sopravvivenza e attività delle cellule osteoblastiche (36-38).

\section{FRATTURE}

I pazienti uremici cronici sono a rischio di fratture con una prevalenza di fratture vertebrali del $21 \%$ e rischio relativo di frattura dell'anca incrementato da 2 a 14 volte rispetto alla popolazione di controllo. Dopo trapianto renale il rischio incrementa con casistiche che riportano un'incidenza di fratture variabile dal $5 \%$ al $44 \%$ dei pazienti. È stato calcolato un incremento di circa 4 volte il rischio di frattura dal periodo pre-trapianto al periodo post-trapianto (0.009 per paziente/anno vs 0.032 per paziente/anno rispettivamente). Nei pazienti trapiantati con diabete mellito l'incidenza di fratture è ancora più alta: interessa circa il 40-49\% dei pazienti $(39,40)$. Gli eventi fratturanti determinano problemi di trattamento (terapia chirurgica e medica), di riabilitazione, sono causa di comorbidità, possono condizionare pesantemente la qualità di vita del paziente trapiantato: è indispensabile pertanto attuare adeguati trattamenti preventivi per ridurre al minimo i fattori di rischio.

1) Osteoporosi definizione: (BV/TV $\%<14 \%)$ : riduzione del $14 \%$ del volume osseo (BV) occupato da tessuto osseo trabecolare calcificato e non calcificato rispetto al volume totale dell'osso (TV).

2) Mineral Lag Time: intervallo tra la deposizione di matrice osteoide e la sua mineralizzazione.

\section{OSTEONECROSI (NECROSI AVASCOLARE)}

Viene definita anche necrosi ischemica o avascolare. La necrosi ossea interessa la testa del femore, ma può colpire anche altri segmenti ossei: la testa e l'epifisi omerale, l'epifisi tibiale, le ossa carpali ecc. Tale necrosi è secondaria a un ridotto flusso sanguigno endomidollare con ischemia conseguente del tessuto osseo: si verifica distruzione del tessuto trabecolare e necrosi cellulare (osteociti e cellule del midollo osseo). La patogenesi non è ben chiarita: attualmente si ritiene che un aumento del tessuto adiposo a livello del midollo (per proliferazione cellulare) incrementando la pressione endomidollare, determinerebbe un ridotto flusso sanguigno nei sinusoidi intramidollari. La durata dell'età dialitica, associata a una terapia steroidea protratta a lungo, con dosi elevate, sono state imputate avere un ruolo prevalente nel determinismo del quadro osteonecrotico. Alcuni studi associano un'aumentata incidenza di questa patologia all'impiego della ciclosporina. Il rischio di tale patologia ossea è andato progressivamente riducendosi negli ultimi anni (incidenza del $15 \%$ negli anni scorsi): in uno studio più recente che ha interessato oltre 40000 pazienti trapiantati renali, l'incidenza di ospedalizzazione dovuta a osteonecrosi è stata di 7 episodi/1000 pazienti trapiantati/anno (41). La risonanza magnetica è la tecnica radiologica più sensibile e andrebbe eseguita in ciascun paziente che presenta dolore persistente alle ginocchia, alle spalle e alle anche. Il trattamento è chirurgico e spesso prevede, se interessata la testa del femore, l'intervento di protesi (42).

\section{Profilassi e trattamento dell'osteopatia post-trapianto}

Poiché la perdita di massa ossea si verifica, come già accennato, nei primi mesi post-trapianto, è indispensabile instaurare terapie preventive assai precocemente.

E opportuno valutare i vari fattori di rischio pre- e posttrapianto e pianificare una terapia adeguata (Tab. II).

\section{Generalità sulla prevenzione e trattamento della patologia ossea post-trapianto}

Il nefrologo può utilizzare varie strategie terapeutiche per prevenire e trattare l'osteopatia del paziente trapiantato renale. I principali supporti terapeutici utilizzati sono: la vitamina $\mathrm{D}$, i difosfonati, i supplementi di calcio.

\section{VITAMINA D}

La vitamina $\mathrm{D}$ e i suoi analoghi sono in grado di ridurre gli effetti osteopenizzanti dei glucocorticoidi, mediante un aumentato assorbimento di calcio (intestinale e renale) e una riduzione della secrezione del PTH. Va tenuto 


\section{TABELLA II - PROFILASSI E TRATTAMENTO DELL'OSTEOPATIA POST-TRAPIANTO}

\section{1) Personalizzare la terapia immunosoppressiva}

Somministrare steroidi al più basso dosaggio compatibile e a emivita breve

2) Evitare diuretici dell'ansa - Somministrare tiazidici nei pazienti ipercalciurici

3) Trattamento del persistente ipogonadismo e ipotiroidismo

\section{4) Adeguata attività fisica - Evitare il fumo}

\section{5) Trattare l'iperparatiroidismo persistente}

Somministrazione di calcio e vitamina D (IPT non ipercalcemico)

Somministrazione dei calciomimetici

Paratiroidectomia

\section{6) Trattare le altra cause di ipercalcemia}

\section{7) Trattare l'ipofosfatemia persistente}

8) Vitamina D: colecalciferolo o ergocalciferolo

Composti attivi della vitamina $\mathrm{D}$

\section{5) Supplementi di calcio}

\section{0) Adeguato apporto nutrizionale}

\section{1) Calcitonina}

12) Difosfonati

presente il rischio di una ipercalcemia e ipercalciuria sulla funzione renale.

In pazienti con buona funzione renale e senza una ipercalcemia persistente, basse dosi di colecalciferolo o ergocalciferolo (600-800 unità/die) possono avere benefici effetti preventivi riducendo i rischi dell'osteopenia da steroidi. Sono consigliabili in alternativa piccole dosi di derivati attivi della vitamina $\mathrm{D}$ : alfacalcidolo, doxercalciferolo (non in commercio in Italia) o calcitriolo (0.25-0.5 $\mathrm{mg}$ /die o a gg. alterni). In particolare la somministrazione di alfacalcidolo in pazienti trattati con steroidi ha prevenuto la demineralizzazzione ossea e l'incidenza di fratture. In uno studio in doppio cieco controllato vs placebo la somministrazione di calcitriolo associato a supplementi di calcio ha ridotto la perdita di massa ossea post-trapianto (43-45). In uno studio recente, pazienti trapiantati renali trattati con vitamina $D$ e supplementi di calcio presentavano, dopo 6 mesi, un incremento della BMD rispetto a un gruppo di pazienti di confronto, senza terapia, nei quali era presente un decremento costante nel tempo della BMD (46). Per contro Wissing ha presentato recentemente un lavoro mostrando che l'aggiunta di vitamina $\mathrm{D}$ al calcio in pazienti trapiantati renali trattati con basse dosi di steroidi non determinava alcun effetto positivo aggiuntivo sulla mineralizzazione ossea (47).

\section{SUPPLEMENTI DI CALCIO}

Il trattamento con steroidi riduce l'assorbimento intestinale di calcio: in presenza di una calcemia normale è consigliabile, pertanto, un apporto di calcio di almeno 1 
$\mathrm{g} /$ die per i maschi, e di almeno $1.5 \mathrm{~g}$ /die per le femmine in post-menopausa. Se i pazienti sono in trattamento con diuretici è preferibile somministrare i tiazidici rispetto alla furosemide che ha una potente azione calciurica. I tiazidici sono come noto, diuretici ipocalciurici; va tenuto comunque presente che il trattamento a lungo termine con tiazidici può determinare alterazioni del metabolismo lipidico (iperlipidemia) e minerale (ipokaliemia).

\section{DIFOSFONATI}

E un dato consolidato che i difosfonati sono assai efficaci nella prevenzione e trattamento della patologia ossea osteoporotica indotta dagli steroidi. Essi agiscono favorendo l'apoptosi osteoclastica, riducendo il riassorbimento osteoclastico e secondariamente il turnover osseo. Diversi studi in letteratura testimoniano gli effetti benefici dei difosfonati anche nei trapiantati renali: sono stati somministrati nell'immediato post-trapianto o successivamente, e hanno preservato e in alcuni casi addirittura migliorato la mineralizzazione scheletrica di questi pazienti (48-51). Tali dati sono stati confermati in una metanalisi di Mitterbauer (52) che ha valutato 5 studi e 180 pazienti trapiantati: $i$ difosfonati somministrati precocemente entro i primi 6-12 mesi dal trapianto hanno consentito di prevenire la perdita di massa ossea.

Recentemente alcuni autori hanno evidenziato una diretta nefrotossicità dei difosfonati (risedronato), ma va detto che questa si verifica solo quando sono usati ad alte dosi in pazienti oncologici. Se usati alle dosi raccomandate, con le opportune precauzioni, in caso di riduzione del VFG, la nefrotossicità diventa un problema di scarsa valenza clinica. Rimangono ancora molte incertezze sulla dose, la via e i tempi più appropriati di somministrazione e sulla loro capacità nel prevenire gli eventi fratturanti. Quest'ultimo dato sembrerebbe, a una prima valutazione superficiale, contrastare con la evidenza consolidata degli effetti positivi dei difosfonati sulla BMD. A questo proposito è opportuno fare alcune considerazioni:

- L'osteoporosi post-menopausale differisce dalla osteoporosi post-trapianto?

Nella classica osteoporosi gli eventi fratturanti interessano in primo luogo lo scheletro assiale, mentre nell'osteoporosi post-trapianto interessano anche lo scheletro appendicolare (piedi, anche).

- I trapiantati renali, come già accennato, spesso presentano una patologia ossea a basso turnover e la somministrazione dei difosfonati che inibiscono il rimodellamento osseo può alterare l'integrità meccanica dell'osso. Recentemente il National Institute of Health ha definito cosi l'osteoporosi: "Malattia scheletrica caratterizzata da una alterata resistenza ossea che predispone a un aumentato rischio di fratture”. La resistenza dell'osso prevede l'integrazione di due componenti fondamentali: la densità dell'osso (determinata dal picco di massa ossea e dalla perdita di massa ossea: la cosiddetta BMD facilmente misurabile con la DEXA) e la qualità dell'osso che comprende varie componenti, difficilmente misurabili, quali: la micro e macroarchitettura ossea, il turnover e la cosiddetta physical composition of bone. Ciò può spiegare il motivo per cui i difosfonati pur favorendo un incremento della BMD non sono in grado di ridurre l'incidenza di eventi fratturanti, in quanto non in grado di agire sulla "qualità dell'osso" (53-54). A conferma di ciò Coco (55) in uno studio su 72 pazienti trapiantati renali che random assumevano pamidronato e vitamina $\mathrm{D}$ più calcio o solo vitamina $\mathrm{D}$ e calcio, ha evidenziato a sei mesi, mediante indagine bioptica, che tutti i pazienti in trattamento con pamidronato presentavano una malattia adinamica dell'osso, vs solo il $50 \%$ dei pazienti nel gruppo di controllo. L'osso adinamico è ad alto rischio di eventi fratturanti, ne deriva che l'utilizzo dei difosfonati nel trattamento dell'osteopatia post-trapianto va valutato caso per caso.

- A conferma di quanto detto, una review sistematica di trial controllati randomizzati, comprensiva di 1209 pazienti trapiantati, effettuata allo scopo di comprendere l'efficacia di diverse strategie terapeutiche (difosfonati o vitamina $\mathrm{D}$ plus or minus calcio, e calcitonina), nel prevenire l'osteopatia, ha dato i seguenti risultati:

- Nessuno studio ha dimostrato una riduzione del rischio di frattura.

- I pazienti che assumevano difosfonati, analoghi della vitamina $\mathrm{D}$, o calcitonina presentavano un miglioramento della densità minerale ossea nei confronti di pazienti senza terapia (56).

\section{Considerazioni finali sull'utilizzo dei difosfonati}

I difosfonati pertanto, non dovrebbero essere usati:

1) in presenza di una patologia ossea a basso turnover (malattia adinamica dell'osso) in quanto potrebbero peggiorare la mineralizzazione ossea;

2) quando il filtrato si riduce al di sotto di $40 \mathrm{~mL} / \mathrm{min} / \mathrm{m}^{2}$;

3) nelle donne in pre-menopausa.

Il loro utilizzo sarebbe consigliato in presenza di una severa osteopenia, in pazienti diabetici, in donne in post-menopausa con un quadro osteoporotico severo ben documentato e in pazienti a grave rischio di frattura o con pregresse fratture soprattutto nei primi 6-12 mesi post-trapianto.

Da quanto detto, emerge la necessità, nel trattamento dell'osteopatia del trapiantato, di avere a disposizione farmaci che stimolino il rimodellamento osseo. 
A questo proposito, nei pazienti con osteoporosi non secondaria a trapianto, sono stati utilizzati potenti farmaci ad azione anabolica sull'osso: il sodio fluoruro e il PTH umano ricombinante. Il primo è altamente efficace nell'aumentare la BFR, la BMD e in alcuni studi, nel ridurre l'incidenza di eventi fratturanti. Purtroppo nei casi di osteoporosi complicata tale farmaco può avere un effetto paradosso, aumentando il rischio di frattura parallelamente all'incremento della BMD.

Il PTH umano ricombinante, somministrato a basso dosaggio, sufficiente ad aumentare la concentrazione dei livelli plasmatici di PTH di 2-3 volte, si è dimostrato un potente agente anabolico, capace di incrementare la $\mathrm{BMD}$ e ridurre il rischio di frattura nelle pazienti con osteoporosi post-menopausale. La somministrazione di tale ormone, nel trattamento della osteoporosi secondaria a steroidi ha mostrato un miglioramento della $\mathrm{BMD}$, un aumento del turnover osseo, ma l'effetto positivo sul- l'incidenza delle fratture è limitato a poche segnalazioni in letteratura. Qualora in un prossimo futuro si dimostrasse una sua reale efficacia nel prevenire gli eventi fratturanti, potrebbe rappresentare per il nefrologo, nonostante i costi molto elevati, un presidio terapeutico aggiuntivo (57-59).

Indirizzo degli Autori:

Dr. Sandro Bandini

SOD Nefrologia dei Trapianti e Dialisi

Azienda Ospedaliera Universitaria Careggi

viale Pieraccini 18

50100 Firenze

sandro.bandini1@virgilio.it

\section{Bibliografia}

1. Canalis E. Clinical Review 83: Mechanism of glucocorticoid action in bone: implications to glucocorticoid -induced osteoporosis. J Clin Endocrinol Metab 1996; 81: 3441-7.

2. Almond MK, Kwan JT, Evans K, Cunningham J. Loss of regional bone mineral density in the first 12 months following renal transplantation. Nephron 1994; 66: 52-7.

3. Ramsey-Goldman R, Dunn JE, Dunlop DD, et al. Increased risk of fracture in patients receiving solid organ transplants. J Bone Miner Res 1999; 14:456-63.

4. Heaf JG. Bone disease after renal transplantation. Transplantation 2003; 75: 315-20.

5. Parfitt AM. Hypercalcemic hyperparathyroidism following renal transplantation: differential diagnosis, management and implications for cell population control in the parathyroid gland. Miner Electrolyte Metab 1999; 8: 92-112.

6. Rix M, Lewin E, Olgaard K. Post transplant bone disease. Transplantation Reviews 2003; 17: 176-86.

7. Lewin E. Involution of the parathyroid glands after renal transplantation. Curr Opin Nephrol Hypertens 2003; 12: 363-71.

8. Traindl O, Langle F, Reading S, et al. Secondary hyperparathyroidism and acute tubular necrosis following renal transplantation. Nephrol Dial Transplant 1993, 8: 173-6.

9. Messa PG, Sindici C, Cannella G. Persistent hyperparat- hyroidism after renal transplantation. Kidney Int 1998; 54: 1704-13.

10. Montalban M, De Francisco AL, Marinoso Ml, Zubimendi $\mathrm{JA}$, Arias M. Bone disease in long term adult kidney transplant patients with normal renal function. Kidney Int 2003; 85(Suppl): S1129 -32.

11. Lindberg JS, Moe SM, Goodman WG, et al. The calcimimetic AMG 073 reduces parathyroid hormone and calcium $\mathrm{x}$ phosphorus in secondary hyperparathyroidism. Kidney Int 2003; 63: 248-54.

12. Srinivas T, Schold J, Kea W. Improvement in hypercalcemia with cinacalcet after kidney tranplantation. Clin J Amer Soc Nephrol 2006; 1: 323-6.

13. Serra AL, Savoca R, Huber AR. Effective control of persistent hyperparathyroidism with cinacalcet in renal allografts recipients. Nephrol Dial transplant 2007; 22: 577-83.

14. Schwarc I, Argiles A, Garrigue V. Cinacalcet chloride is efficient and safe in renal transplant recipients with posttransplant hyperparathyroidism.Transplantation 2006; 82: 675-82.

15. Kruse AE, Eisenberger U, Felix JF, Mohaupt MG. Effect of cinacalcet cessation in renal transplant recipients with persistent hyperparathyroidism. Nephrol Dial Transplant 2007; 22: 2362-5.

16. D’Alessandro AM, Melzer JS, Gillen DL, et al. Tertiary hyperparathyroidism after renal transplantation: operative indications. Surgery 1989; 106: 1049-55.

17. Schwarz A, Rustien G, Mrkel S, Radermacher J, Haller H. 
Decreased renal function after parathyroidectomy. Nephrol Dial Transplant 2007; 22: 584-91.

18. Levi M. Post transplant hypophosphatemia. Kidney Int 2001; 59: 2377-87.

19. Green J, Debby H, Lederer E, Levi M, Zajicek HK, Bick T. Evidence for a PTH independent humoral mechanism in post transplant hypophosphatemia and phosphaturia. Kidney Int 2003; 64: 2272-9.

20. Pande S, Ritter CS, Rothstein M. FGF - 23 and sFRP-4 in chronic kidney disease and post renal transplantation. Nephron Physiol 2006; 104: 23-8.

21. Bhan I, Shah A, Holmes J. Post transplant hyposphatemia “Hyper - phosphatoninism?”. Kidney Int 2006; 70: 1486-91.

22. Balal M, Paydas S, Seyrek N . Dipyridamole for renal phosphate leak in successfully renal transplant hypophosphatemic patients. Clin Nephrol 2005; 63: 87-90.

23. Brandenburg MV, Westenfeld R, Ketteler M. The fate of bone after renal transplantation. J Nephrol 2004; 17: 190-204.

24. Rubin R, Bilezekian JP. Clinical review151: The role of parathyroid hormone in the patogenesis of glucocorticoidinduced osteoporosis: a re-examination of the evidence. J Clin Endocrinol Metab 2002; 87: 4033-441.

25. Julian BA, Lasow DA, Dubovsky J, Curtis JJ, Quarles LD. Rapid loss of vertebral mineral density after renal transplantation. N Engl J Med 1991; 325: 544-50.

26. Patel S, Kwan JT, McCloskey E, et al. Prevalence and causes of low bone density and fractures in kidney tranplants patients. J Bone Miner Res 2001; 16: 1863-70.

27. Moe SM. The treatment of steroid bone loss in transplantation. Curr Opin Nephrol Hypertens 1997; 6: 544-9.

28. Epstein S. Post-transplantation bone disease: the role of immunosuppressive agents and the skeleton. J Bone Miner Res 1996; 11: 1-7.

29. Wenstein RS. Glucocorticoid-induced osteoporosis. Rev Endocr Metab Disord 2001; 2: 65-73.

30. Hofbauer LC, Gori F, Riggs BL, Lacey DL, Dunstan CR, Spelsberg TC , Khosla S. Stimulation of osteoprotegerin ligand and inhibition of osteoprotegerin production by glucocorticoids in human osteoblastic lineage cells: Potential paracrine mechanism of glucocorticoid -induced osteoporosis. Endocrinology 1999; 140: 4382-9.

31. Stewart PJ, Stern PH. Cyclosporines: correlation of immunosoppressive activity and inhibition of bone resorption. Calcif Tissue Int 1989; 45: 222-6.

32. Westeel FP, Mazouz H, Ezaitouni F, et al. Cyclosporine bone remodeling effect prevents steroid osteopenia after kidney transplantation. Kidney Int 2000; 58: 1788-96.

33. Goffin E, Devogelaer JP, Lalaoui A, et al. Tacrolimus and low-dose steroid immunosuppression preserves bone mass after renal transplantation. Transpl Int 2002; 15: 73-80.

34. Monegal A, Navasa M, Guanabens M, et al. Bone mass and mineral metabolism in liver transplant patients treated with FK506 or cyclosporine. Calcified Tissue Int 2001; 68: 83-6.

35. Bryer HP, Isserow JA, Amstrong EC, et al. Azathioprine alone is bone sparing and does not alter cyclosporin A-induced osteopenia in rat. J Bone Miner Res 1995; 10:132-8.

36. Goodman GR, Dissanayake IR, Sodam BR, et al. Immunosuppressant use without bone loss-implications for bone loss after transplantation. J bone Miner Res 2001; 16: $72-8$.

37. Bellorin-Font E, Rojas E Carlini RG, Subiaga O, Weisinger Jr. Bone remodeling after renal transplantation. Kidney Int 2003; (Suppl): S125-8.

38. Cueto-Manzano AM, Konel S, Hutchinson AJ, et al. Bone loss in long term transplantation: histopathology and densitometry analysis. Kidney Int 1999; 55: 2021- 9.

39. Alem AM, Sherrard DJ, Gillen DL, et al. Increased risk of hip fracture among patients with end stage renal disease. Kidney Int 2000; 58: 396-9.

40. Nisbeth U, Lindh E, Ljunghall S, Backman U, Fellstrom B. Increased fracture rate in diabetes mellitus and female after renal transplantation. Transplantation 1999; 67: 1218- 22 .

41. Abbott KC, Oglesby RJ, Bohen M. Hospitalized avascular necrosis after renal transplantation in the United States. Kidney Int 2002; 62: 2259-2256.

42. Lausten GS, Lemser T, Jensen PK, Egfijord M. Necrosis of the femoral head after kidney transplantation. Clin Transplant 1998; 12: 572-4.

43. Josephson MA, Schumm LP, Chiu MY, Marshall C, Thristlethwaite JR, Sprague SM. Calcium and calcitriol prophylaxis attenuates posttranplant bone loss. Transplantation 2004; 78: 1233-6.

44. Ringe JD, Coster A, Meng T, Schacht E, Umbach R. Treatment of glucocorticoid-induced osteoporosis with alfacalcidol/calcium versus vitaminD/calcium. Calcif Tissue Int 1999; 65: 337-40.

45. Reginster JY, Lecart MP, Sarlet N, Defraigne JO. Alphacalcidol in prevention of glucocorticoid-induced osteoporosis. Calcif Tssue Int 1999; 65: 328-31.

46. De Sevaux RG, Hoitsma AJ, Corstens FH, Wetzels JF. Treatment of vitamin $\mathrm{D}$ and calcium reduces bone loss after renal transplantation: a randomized study. J Am Soc Nephrol 2002; 13: 1608-12.

47. Wissing KM, Broeders N, Moreno Reyes R. A controlled study of vitamin $\mathrm{D} £$ to prevent bone loss in renal transplant patients receiving low doses of steroids. Transplantation 2005; 79: 108-12.

48. Roux C, Oriente P, Laan R, et al. Randomized trial of effect of cyclical etidronate in the prevention of corticosteroid - induced bone loss. J Clin Endocrinol Metab 1998; 83: 1128-33.

49. Haas M, Leko-Mohr Z, Roschger P, et al. Zoledronic acid 
to prevent bone loss in the first 6 months after renal transplantation .Kidney Int 2003; 63: 1130-6.

50. Arlen DJ, Lambert K, Ioannidis G, Adachi JD. Treatment of established bone loss after renal transplantation with etidronate.Transplantation 2001; 71: 669-73.

51. Cunningham J, Sprague SM, Cannata Andia. Osteoporosis in chronic kidney disease: Am J kidney Dis 2004; 43: 566-71.

52. Mitterbauer C, Schwarz C, Haas M, Oberbauer R. Effects of bisphosphonates on bone loss in the first year after renal transplantation - a meta-analysis of randomized controlled trial. Nephrol Dial Transplant 2006; 21: 2275-81.

53. Weber TJ, Quarles LD. Preventing bone loss after renal transplantation with bisphosphonates: We can... but should we ? Kidney Int 2000; 57: 735-8.

54. Cunningham J. Bisphosphonates in the renal patient. Neprhol Dial Transplant 2007; 22: 1505-7.
55. Coco M, Glicklich D, Faugere MC, et al. Prevention of bone loss in renal transplant recipients: a prospective randomized trial of intravenous pamidronate. J Am Soc Nephrol 2003; 14: 2669-76.

56. Palmer SC, Strippoli GF, Mc Gregor DO. Interventions of preventing bone disease in kidney tranplant recipients. Cochrane Database Syst R 2 : CD005015, 2005.

57. Chen P, Miller PD, Delmas PD. Change in Lumbar spine BMD and Vertebral Fracture Risk Reduction in Teriparatide-Treated Postmenopausal Woman With osteoporosis. J Bone Miner Res 2006; 17: 273-7.

58. Nevitt MC, Chen P, Dore RK. Reduced risk of back pain following teriparatide treatment: a meta-analysis. Osteoporos Int 2006; 17: 273-9.

59. Black DM, Bilezikian JP, Ensrud KE. One year of alendronate after one year of parathyroid hormone (1-84) for osteoporosis. N Engl J Med 2005; 353: 555-62. 